assessed and blood and urine samples were collected to test for STIs [Chlamydia trachomatis (CT), Neisseria gonorrhoeae (NG), syphilis and HIV]. Analysis at both the aggregate and state level was done. Adjusted ORs were obtained by including all socio-demographic variables associated with rounds of survey in multivariate models. Results At the aggregate level, among FSWs, prevalence of syphilis declined from $10.8 \%$ in $\mathrm{R} 1$ to $5.0 \%$ in $\mathrm{R} 2$ (AOR 0.39, $\mathrm{p}=0.001$ ) and no significant change was observed in prevalence of HIV, NG or CT. Among FSW clients, STI prevalence was low in R1 (syphilis $4.7 \%$, NG $0.4 \%$ and CT $2.4 \%$ ) and no significant change was observed in R2. The state-wide trend shows that for FSWs in AP, a significant decline occurred in prevalence of both HIV (AOR 0.68, p<0.01) and syphilis (AOR $0.39, p<0.001$ ) whereas in $\mathrm{TN}$, only syphilis prevalence declined (AOR 0.20, $\mathrm{p}<0.001$ ). The trend in $\mathrm{MH}$ differs from the other two states. In $\mathrm{MH}$, prevalence of bacterial STIs (syphilis, NG and CT) declined significantly whereas HIV prevalence increased significantly (AOR 1.29, $\mathrm{p}=0.04$ ). Among FSW clients, STI prevalence was low in $\mathrm{MH}$ (syphilis $4.3 \%$, NG $0.7 \%$, and CT $4.2 \%$ ) and TN (syphilis $4.1 \%$, NG $0.0 \%$, and CT $0.8 \%$ ) in R1 and remained unchanged in R2. However, a significant decline occurred in HIV prevalence in both states [MH: AOR-0.25, $p=0.002$; TN: AOR-2.3, $\mathrm{p}<0.05]$. In $A P$, only prevalence of syphilis (AOR $0.18, p<0.001$ ) declined and no change was observed in other STIs.
Conclusion The prevalence of syphilis declined among both FSWs and their clients in all three states whereas no change was seen in other bacterial STI prevalence (NG or CT). Among FSW clients in $\mathrm{MH}$ and TN, STI prevalence remained unaltered. Tailored and sustained programmatic efforts are needed to address the disparity in prevalence of STIs and HIV in the three states.

\section{1-S08.06 VARIABILITY IN THE NUMBERS AND TYPES OF SEX PARTNERS OF FEMALE SEX WORKERS AND ITS IMPACT ON HIV PREVALENCE IN SOUTHERN INDIA}

doi:10.1136/sextrans-2011-050109.48

${ }^{1} \mathrm{~K}$ Deering, ${ }^{1} \mathrm{~J}$ Shoveller, ${ }^{2} \mathrm{M}$ Pickles, ${ }^{3} \mathrm{~S}$ Shaw, ${ }^{3} \mathrm{~S}$ Moses, ${ }^{3} \mathrm{~J}$ Blanchard, ${ }^{4} \mathrm{R}$ Washington, ${ }^{4} \mathrm{~B} \mathrm{M}$ Ramesh, ${ }^{4} \mathrm{~S}$ Isac, ${ }^{2} \mathrm{M} \mathrm{C}$ Boily. ${ }^{1}$ University of British Columbia, Vancouver, Canada; ${ }^{2}$ Imperial College, UK; ${ }^{3}$ University of Manitoba, Winnipeg, Canada; ${ }^{4}$ Karnataka Health Promotion Trust, Bangalore, India

Background Heterogeneity in the structure of sex work within and across female sex worker (FSW) populations may be an important determinant of the spread of HIV. We characterised heterogeneity in sexual structure and assessed how it influenced HIV prevalence on a population level in southern India.

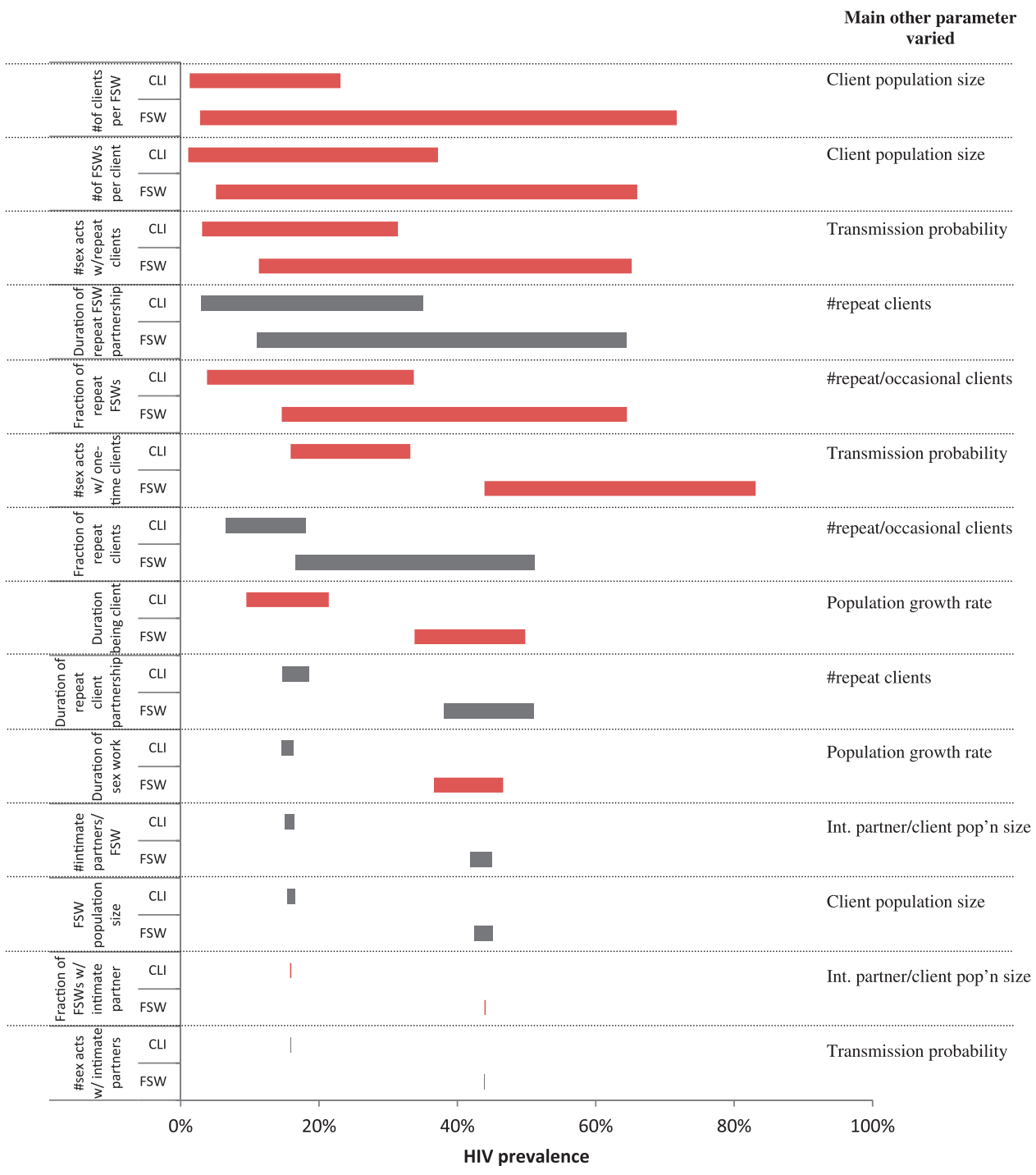

Abstract 01-S08.06 Figure 1 Impact of varying each sexual structure parameter through a wide range (half to double the baseline value) on peak HIV prevalence in district A. . ${ }^{1}$ Red bars=a positive correlation and grey bars=a negative correlation. Bars are arranged in the order of the absolute impact of each parameter on peak HIV prevalence. 
Methods We used data collected from FSWs and clients in three districts (A, B, C) in Karnataka state, India, as part of the Avahan AIDS Initiative, a large-scale targeted HIV prevention program. A deterministic model of HIV transmission among FSWs/clients was parameterised/fitted to the district (A) with the highest FSW/client HIV prevalence in 2005 (33.9\%/6.2\%). We conducted multiple sensitivity analyses to examine how sexual structure parameters (Abstract 01-S08.06 figure 1) influenced modelled HIV prevalence within district $\mathrm{A}$ and identified sources of heterogeneity that could explain differences in HIV prevalence between district $\mathrm{A}$ and districts B and C.

Results Duration of sex work (2.6-fold), duration of the repeat client/FSW relationship (1.7-fold) and numbers of clients of FSWs (1.6-fold) exhibited the largest differences across districts. In district A, doubling the numbers of clients of FSWs, numbers of visits to FSWs by clients and frequency of sex acts with repeat clients increased HIV prevalence the most. Interestingly, doubling the duration of the repeat FSW-client relationship and fraction of repeat clients decreased HIV prevalence the most (Abstract O1-S08.06 figure 1). The observed differences in the mean numbers of clients per month between districts (A:56; B:46; C:34) could alone explain most of the lower HIV prevalence in districts $B$ and $C$ relative to district $\mathrm{A}$. Relative to that observed in district $\mathrm{A}$, the lower number of sex acts with repeat clients in district B and larger FSW population size in district $\mathrm{C}$ could also explain these districts' lower HIV prevalence.

Conclusions The impact of each sexual structure parameter on HIV prevalence depended on the values of other parameters, since many parameters were correlated to keep the total number of FSW-client/ client-FSW partnerships equal. Although the rules defining how parameters need to be empirically validated, results demonstrate how their definition were of critical importance in influencing outcomes. The heterogeneous risk faced by FSWs needs to be recognised to explain differences in HIV prevalence, and to inform population-specific interventions capable of addressing the complexities implied by this heterogeneity.

\section{Epidemiology oral session 9: Use of observa- tional data and mathematical modelling for impact evaluation \\ 01-S09.01 INCREASED CONDOM USE AND DECREASED HIV \& STI PREVALENCE AMONG FEMALE SEX WORKERS FOLLOWING A TARGETED HIV PREVENTION PROGRAM IN KARNATAKA, SOUTH INDIA}

doi:10.1136/sextrans-2011-050109.49

\begin{abstract}
${ }^{1} \mathrm{~S}$ Moses, ${ }^{2} \mathrm{~B}$ M Ramesh, ${ }^{3} \mathrm{~S}$ Isac, ${ }^{1} \mathrm{~S}$ Reza-Paul, ${ }^{4} \mathrm{M}$ Alary, ${ }^{4} \mathrm{~J}$ Bradley, ${ }^{2} \mathrm{R}$ Washington, ${ }^{5} \mathrm{~T}$ Beattie, ${ }^{1} \mathrm{~J}$ Blanchard. ${ }^{1}$ University of Manitoba, Winnipeg, Canada; ${ }^{2}$ Karnataka Health Promotion Trust, Bangalore, India; ${ }^{3} \mathrm{KHPT}$, Bangalore, India; ${ }^{4}$ Centre hospitalier affilié universitaire de Québec, Quebec, Canada; ${ }^{5}$ London School of Hygiene and Tropical Medicine, London, UK
\end{abstract}

Background We assessed the impact of a large-scale, targeted HIV prevention program among female sex workers (FSWs) in Karnataka state, south India.

Methods In the context of an HIV prevention program involving 18 districts, funded by the Bill \& Melinda Gates Foundation, we conducted initial (R1) and two follow-up (R2 and R3) survey rounds (integrated behavioural and biological assessments), involving random samples of urban FSWs in three selected districts. The total urban FSW population size of the three districts is estimated at 8600. Initial surveys were conducted at 7-19 months following program commencement in 2004-2005, and each follow-up survey at two to three year intervals thereafter. Multivariable logistic regression models were used, with weights to account for a complex sampling design. Results presented are for the three districts combined.

Results 1242 FSWs participated in R1, 1238 in R2 and 1248 in R3. In R3, $98 \%$ and $92 \%$ of FSWs reported contact by a peer educator and having visited a project STI clinic, respectively. HIV prevalence declined steadily from R1 to R3, and in R3 compared to R1, there were reductions in HIV prevalence $(24.8 \%$ vs $13.3 \%$, adjusted OR $(\mathrm{AOR})=0.59,95 \%$ CI $0.41 \%$ to $0.86 \%, \mathrm{p}=0.005)$; active syphilis $(12.7 \%$ vs $8.8 \%, \mathrm{AOR}=0.44,95 \% \mathrm{CI} 0.31 \%$ to $0.61 \%, \mathrm{p}<0.001)$; and high-titre syphilis $(6.6 \%$ vs $3.2 \%, \mathrm{AOR}=0.33,95 \%$ CI $0.21 \%$ to $0.51 \%, \mathrm{p}<0.001)$. Trichomoniasis (Tv), chlamydial infection $(\mathrm{Ct})$ and gonorrhoea $(\mathrm{Ng})$ were assessed in one district in R3. From R1 to R3, Tv declined from $32.9 \%$ to $3.5 \%$ ( $\mathrm{AOR}=0.07$, 95\% CI $0.04 \%$ to $0.15 \%, \mathrm{p}<0.001$ ); $\mathrm{Ng}$ from $5.4 \%$ to $0.9 \%$ (AOR $0.22,95 \%$ CI $0.0 .07 \%$ to $0.71 \%, p=0.01$ ); and Ct from $10.8 \%$ to $4.6 \%$ (AOR $0.38,95 \%$ CI $0.19 \%$ to $0.75 \%, p=0.006)$. Comparing $\mathrm{R} 3$ with $\mathrm{R} 1$ in all three districts, reported condom use at last sex increased significantly for repeat clients $(60.1 \%$ to $93.9, \mathrm{AOR} 11.2,95 \%$ CI $7.2 \%$ to $17.5 \%$, $\mathrm{p}<0.001)$; for occasional clients $(81.0 \%$ to $88.2 \%$, AOR $2.8,95 \% \mathrm{CI}$ $1.9 \%$ to $4.1 \%, \mathrm{p}<0.001$ ); and for regular (non-commercial) partners (24.4\% to $55.9 \%$, AOR $5.0,95 \%$ CI $2.4 \%$ to $10.3 \%, p<0.001$ ) Condom use overall was strongly associated with level of peer educator contact, having been exposed to condom demonstrations, and attendance at a program health clinic.

Conclusions Over 6 years, this large-scale HIV prevention program for FSWs achieved high rates of program coverage, high rates of condom use with clients, and significant reductions in HIV and STI prevalence. It will be important to sustain these efforts in the long term.

\section{1-S09.02 HIV ELIMINATION WITHOUT ANTIRETROVIRAL THERAPY (ART) IN SOUTHERN INDIA: MODELLING AND PROJECTED COSTS}

doi:10.1136/sextrans-2011-050109.50

${ }^{1} \mathrm{M}$ Pickles, ${ }^{2} \mathrm{P}$ Vickerman, ${ }^{2} \mathrm{~A}$ Vassall, ${ }^{3} \mathrm{BM}$ Ramesh, ${ }^{4} \mathrm{~K}$ Deering, ${ }^{2} \mathrm{~S}$ Chandrashekar, ${ }^{3} \mathrm{~S}$ Isac, ${ }^{3} \mathrm{~S}$ Moses, ${ }^{5} \mathrm{M}$ Alary, ${ }^{1} \mathrm{M} \mathrm{C}$ Boily. ${ }^{1}$ Imperial College London, UK; ${ }^{2}$ LSHTM, UK; ${ }^{3}$ KHPT, India; ${ }^{4}$ University of Manitoba, Winnipeg, Canada; ${ }^{5}$ Centre Hospiltalier Affilié Universitaire de Québec, Quebec, Canada

Background There is widespread interest in the use of antiretroviral therapy for eliminating HIV. However, it is possible that bringing other existing interventions to scale, such as condom promotion among female sex workers (FSWs) could reduce HIV transmission to low levels in some settings. Avahan, the Indian AIDS Initiative, has attained high coverage among FSWs in southern India, achieved large increases in condom use (>90\% use condoms consistently), and substantial reductions (35-60\% relative decreases over 5 years) in FSW HIV prevalence have been observed in the three districts with three rounds of FSW surveys. This modelling analysis projects the long-term impact of the Avahan intervention strategy in these districts.

Methods A bespoke dynamical model of HIV transmission among FSW/clients was parameterised and fitted to district specific behavioural and STI/HIV prevalence data within a Bayesian framework. The model fits for each district were used to determine whether the long-term impact of the current level of condom use achieved through Avahan, will reduce the HIV incidence among FSWs and clients to less than 1 infection per 1000 FSWs and clients per year (elimination definition used by Granich, Lancet 2009). The probability of local elimination, time till elimination, and costs (extended from detailed costing for first 3 years in 2008 US\$) and HIV infections averted of achieving this was estimated.

Results With the current high level of condom use in all 3 districts, the probability that the HIV incidence among FSWs and clients becoming $<1$ infection per 1000 by 2050 is $>95 \%$. The Abstract O1- 